( $\mathrm{n}=825 ; 79,5 \%)$ wiederum treten nur vereinzelt oder gar keine Symptome auf. Auffällig war, dass das Risiko der Kinder, an einer $\mathrm{AD}$ vom frühen persitierenden Phänotyp zu erkranken, fünffach erhöht war, wenn beide Eltern Allergiker waren.

Die verschiedenen Phänotypen waren mit unterschiedlichen Risiken für weitere Allergien assoziiert. Bei Kindern, die bereits sehr früh in ihrem Leben erste Hautsymptome zeigten, wurden in den nächsten Jahren deutlich häufiger Nahrungsmittelallergien diagnostiziert als bei Kindern anderer Phänotypen. Beim frühen transienten Phänotyp war das Risiko mehr als dreimal so hoch (Odds Ratio [OR]: 3,71; 95\%-Konfidenzintervall [KI]:
1,66-8,26), beim frühen persistierenden Phänotyp stieg es sogar um mehr als das Siebenfache(OR: 7,79; $95 \%-K I: 3,42-17,73$ ). Auch das Asthmarisiko variierte je nach Phänotyp und war bei Kindern mit früh einsetzenden und persistierenden Symptomen deutlich erhöht (OR: 2,87; $95 \%$-KI: 1,31-6,31). Im Vergleich zum späten Phänotyp lag der Anteil der an Asthma erkrankten Kinder in der Subgruppe des frühen persistierenden Phänotyps mehr als doppelt so hoch (7,5\% vs. 17,5\%). Der frühe persistierende (OR: 4,04; 95\%-KI: 1,82-8,95) sowie der späte Phänotyp (OR: 3,23; $95 \%-K I$ : 1,37-7,61) waren wiederum mit einem erhöhten Risiko für eine allergische Rhinitis assoziiert.
Fazit: Abhängig vom Erkrankungsalter und dem Erkrankungsverlauf lassen sich bei Kindern vier verschiedene AD-Phänotypen unterscheiden. Die verschiedenen Phänotypen seien wiederum mit einem unterschiedlichen Allergierisiko assoziiert, wobei Kinder mit frühem persistierendem Phänotyp besonders allergiegefährdet seien, so die Autoren. Sie hoffen, mit der Identifizierung von Subgruppen wichtige Anhaltspunkte für die Entwicklung wirksamer Präventionsstrategien geliefert zu haben.

Dr. Dagmar Kraus

Roduit C et al. Phenotypes of Atopic Dermatitis Depending on the Timing of Onset and Progression in Childhood. JAMA Pediatr 2017; 171: $655-62$

\title{
Schwere Nahrungsmittelallergien: Lipidtransferproteine wohl auch in Mitteleuropa ein wichtiger Auslöser
}

\section{Die Rolle von Sensibilisierungen gegen Lipidtransferproteine als Ursache für Allergien gegen pflanzliche Lebensmittel in Mitteleuropa ist bisher kaum erforscht. Eine monozentrische Studie nahm sich dieses Themas an und untersuchte auch die Rolle von Pru p 3 bei solchen Allergien.}

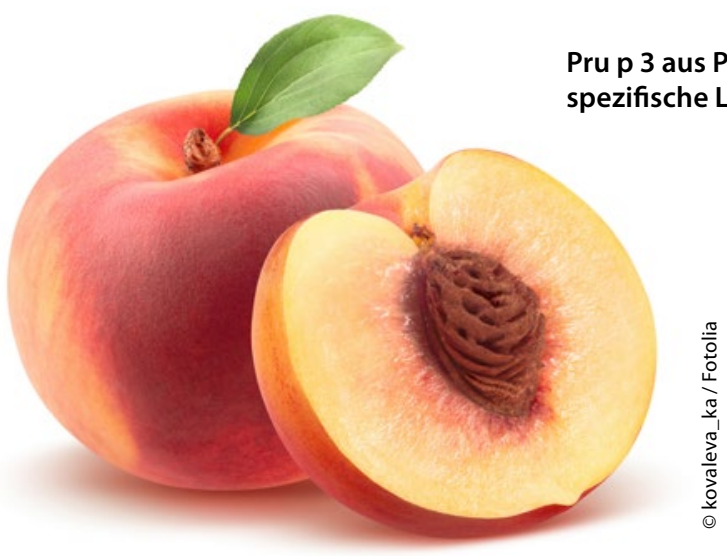

Dis Sensibilisierung gegen nicht spezifische Lipidtransferproteine (nsLTPs) ist vor allem aus dem Mittelmeerraum bekannt und ruft dort vor allem nach dem Genuss von Früchten oder Gemüse teilweise schwere allergische Symptome hervor. Führendes nsLTP-Allergen ist das Pfirsich-LPT Pru p 3, das deshalb auch als Marker für schwere Nahrungsmittelallergien empfohlen wird. Über die Bedeutung einer Pru-p-3-Sensibilisierung bei mittel- und nordeuropäischen
Patienten mit schwerer Allergiesymptomatik ist bisher allerdings nicht viel bekannt - vor allem wegen der schwierigen Abklärung beim Einsatz von Pflanzenextrakten aufgrund von häufigen Kosensibilisierungen gegen Bet v 1 oder kreuzreaktive Profiline.

Monozentrisch wurden nun die spezifischen IgE-Reaktivitäten auf rPru p 3, rBet v 1 und dem Phleum-pratense-Profilin $\mathrm{rPhl}$ p 12 per ImmunoCAP bei 13 österreichischen Patienten untersucht. Bei allen Patienten waren schwere anaphylaktische Reaktionen auf pflanzliche Nahrungsmittel bekannt und alle Patienten zeigten im Hautpricktest bei einer breiten Palette von pflanzlichen Nahrungsmitteln deutliche Quaddelreaktionen mit einem Durchmesser von $8 \mathrm{~mm}$ oder mehr.

Bei zehn der 13 Patienten ergaben sich Sensibilisierungen gegen Pru p 3, bei neun gegen Bet $\mathrm{v} 1$ und bei zwei gegen
Profilin. Damit liegen nun deutliche Hinweise vor, dass auch bei schweren Nahrungsmittelallergien von Mitteleuropäern Sensibilisierungen gegen LPTs eine nicht zu unterschätzende Rolle spielen. Immerhin $60 \%$ der Teilnehmer dieser kleinen Studie waren sowohl Bet-v-1als auch Pru-p-3-positiv.

Die Abklärung dieser LPT-vermittelten Allergien gelingt vermutlich am besten mit der komponentenbasierten Diagnostik („component-resolved diagnostic“, CRD) mit Pru p 3 als Markerprotein. Im Immunoblot war das Lipidtransferprotein in einer ganzen Reihe von rohen und gekochten pflanzlichen Nahrungsmitteln nachweisbar. Wird ein Hautpricktest zur Abklärung benutzt, schlagen die Autoren den Einsatz von gekochten Extrakten vor, denn anders als Bet v 1 ist Pru p 3 hitzestabil.

Fazit: Patienten mit schweren Allergien gegen pflanzliche Nahrungsmittel haben auch in Mitteleuropa häufig eine Sensibilisierung gegen LPTs. Dabei scheint Pru p 3 ein geeignetes Markerprotein für den Sensibilisierungsnachweis $\mathrm{zu}$ sein.

Dr. Barbara Kreutzkamp

Mothes-Luksch N et al. Pru p 3, a marker allergen for lipid transfer protein sensitization also in Central Europe. Allergy 2017; 72: 1415-8 